\title{
Nutrition and the Hallmarks of Aging
}

\author{
K. Virecoulon Giudici \\ Gerontopole of Toulouse, Institute of Aging, Toulouse University Hospital, Université Toulouse III Paul Sabatier, Toulouse, France.
}

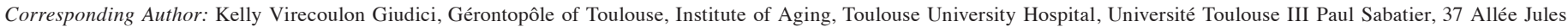
Guesde, 31000 Toulouse, France, E-mail: kellygiudici@gmail.com

Abbreviations: ATP: adenosine triphosphate; DHA: docosahexaenoic acid; EGCG: epigallocatechin gallate; EPA: eicosapentaenoic acid; LINE-1: long interspersed elements; MedDiet: Mediterranean diet; PUFA: polyunsaturated fatty acids.

W ith life expectancy continually increasing, the search for effective treatments able to delay biological aging and to prevent or postpone the development of age-related chronic diseases remains accelerated. In 2013, nine common denominators of the aging process were proposed by López-Otín et al. (1) as the hallmarks of aging: genomic instability, telomere attrition, epigenetic alterations, loss of proteostasis, deregulated nutrient sensing, mitochondrial dysfunction, cellular senescence, stem cell exhaustion, and altered intercellular communication. With increasing attention given to these interconnected cellular and molecular hallmarks and to how they can be controlled, the progressive knowledge on the field points to nutrition as one of the major modifiable factors known to modulate the aging process, shedding light on the therapeutic potential of nutrients, bioactive compounds and, more importantly, of diet as a whole (2-4).

Several nutrients are believed to contribute to a healthy aging. Omega-3 polyunsaturated fatty acids (PUFA) - mainly docosahexaenoic acid (DHA) and eicosapentaenoic acid (EPA) - are anti-inflammatory, capable of reducing oxidative stress (5), and have shown an ability to restore mitochondrial oxidative capacity in different tissues, improving cellular respiration and adenosine triphosphate (ATP) production (6, 7). Folate is an important methyl donor and therefore highly needed for DNA methylation (and consequently for avoiding genomic instability) (8). In this sense, an observational study with young women observed that those with folate deficiency were 3.6 times more likely to show long interspersed elements (LINE-1) hypomethylation in blood leukocytes (9). Vitamin D, in turn, is crucial for a wide range of vital cellular processes including cellular differentiation, proliferation and apoptosis (10). There is also evidence that it might play a role in telomere biology and genomic stability, with an ability to reduce telomere shortening possibly through anti-inflammatory and anti-cell proliferation mechanisms (10).

Using a nontargeted metabolomics method, Tokarz et al. (11) have recently explored in a mouse model how diet and exercise Received September 28, 2021

Accepted for publication September 30, 2021 would impact biological aging, and found a common skeletal muscle metabolic signature of 50 metabolites distinguishing healthy from unhealthy aging. In summary, reduced metabolites from arginine and lysine (two dietary amino acids) and their related metabolic processes were identified as important metabolic hubs of biological aging, mostly associated with oxidative stress and nutrient sensing pathways (11). These findings suggest that maintaining normal arginine and/or lysine levels may be particularly useful to fight sarcopenia and other age-related physiological impairments (11).

A number of bioactive compounds have also been shown to benefit the aging process, mainly due to their antioxidant and anti-inflammatory properties, and also through the modulation of the gut microbiota $(12,13)$. Resveratrol, found mainly in grapes, red wine, peanuts and soy, is one of the most studied polyphenols. It is capable of improving mitochondrial function $(14,15)$, reducing oxidative stress and stimulating autophagy, enhancing the removal of damaging protein misfolding and dysfunctional organelles (15). Oleuropein, oleocanthal and hydroxytyrosol are phenolic compounds found in extra-virgin olive oil related to the protection of proteostasis, reduction of oxidative stress, improvement of mitochondrial function and/or enhancement of hematopoietic stem cell survival and differentiation potential (16-21). Moreover, senolytic activities have been attributed to polyphenols typically found in fruits and vegetables such as epigallocatechin gallate (EGCG) (22-24), quercetin $(2,24)$ and fisetin $(24,25)$. Nevertheless, the adequate dosages that may exert such beneficial effects on the human body are to be defined and, for some compounds, possibly exceed the amounts that could be obtained by eating their natural sources (non-fortified foods) (24).

Diets with a ketogenic profile or the intake of ketogenic supplements (as ketone esters, ketone salts and medium chain triglycerides) have been associated with neuroprotective effects and with positive actions on metabolism, with the potential of delaying the aging process (26). Achieving safe states of ketosis by increasing circulating levels of ketone bodies (mainly $\beta$-hydroxybutyrate) is believed to improve mitochondrial function, to reduce oxidative stress and inflammation, to modulate histone and non-histone acetylation, as well as neurotransmitter systems and RNA functions, through similar mechanisms achieved by caloric restriction $(26,27)$. The current limited number of studies focused on demonstrating such relationships, however, highlights the need of further research on the topic. 
Talking about dietary patterns, the Mediterranean diet (MedDiet) shows a great potential in positively affecting the hallmarks of aging, through multiple mechanisms (3). This pattern adopted by the populations of regions such as Greece, Italy and Spain before the industrial era is still popular (in spite of the increased mixing with the Western dietary pattern), and is mainly composed by vegetables, fruits, legumes, olive oil, fatty fish, grains, nuts and seeds, and by moderate red wine intake (3). These foods are great sources of several aforementioned components, and generally low in sodium, trans fat, sugar and additives. Interestingly, a number of studies were able to observe positive effects of the MedDiet on most of the hallmarks of aging (genomic instability (28-30), telomere attrition (31-34), epigenetic alterations $(35,36)$, stem cell exhaustion $(37,38)$ and altered intercellular communication (39-41)). These findings points to the singularity of the dietary synergism, suggesting that the combination of abundant nutrients and bioactive compounds (and restriction of others) may optimize biological responses in a unique way, that a more simplistic single- or multi-nutrient supplementation approach may not be fully capable of mimicking. However, effects of the MedDiet may vary according to genetic and ethnic characteristics of the studied populations $(33,34)$. It should also be noted that many of these studies were observational. They are howsoever promising, and stimulate further investigation in future randomized controlled trials with large samples.

In times when the run for developing effective and safe medications to fight common age-related degenerative diseases such as Alzheimer's disease and other dementias is still ongoing (42), modifiable lifestyle factors stand out as strong targets towards improving quality of life and favoring a healthy aging trajectory. Current knowledge suggests that the benefits of nutrition on aging are major when interventions are early implemented, during time-sensitive windows before metabolic processes and pathological cascades become irreversible (4, 43). The scientific advances here presented clearly indicates that caring about patients' dietary habits should not be only triggered by the diagnosis of a chronic condition such as diabetes or hypertension, but a basic practice in clinical care, independently of individuals' age. This will only be possible through a more integrated multi-professional outlook, with the assistance of dietitians (among other health professionals) importantly optimizing the regular care of adults and older adults.

Conflicts of interest: None.

\section{References}

1. López-Otín C, Blasco MA, Partridge L, Serrano M, Kroemer G. The hallmarks of aging. Cell. 2013;153(6):1194-217. doi: 10.1016/j.cell.2013.05.039.

2. Russo GL, Spagnuolo C, Russo M, Tedesco I, Moccia S, Cervellera C. Mechanisms of aging and potential role of selected polyphenols in extending healthspan. Biochem Pharmacol. 2020;173:113719. doi: 10.1016/j.bcp.2019.113719.

3. Shannon OM, Ashor AW, Scialo F, Saretzki G, Martin-Ruiz C, Lara J, et al. Mediterranean diet and the hallmarks of ageing. Eur J Clin Nutr. 2021;75(8):11761192. doi: 10.1038/s41430-020-00841-x.

4. Siervo M, Shannon OM, Llewellyn DJ, Stephan BC, Fontana L. Mediterranean diet and cognitive function: From methodology to mechanisms of action. Free Radic Biol Med. 2021;176:105-117. doi: 10.1016/j.freeradbiomed.2021.09.018.

5. Djuricic I, Calder PC. Beneficial Outcomes of Omega-6 and Omega-3 Polyunsaturated Fatty Acids on Human Health: An Update for 2021. Nutrients. 2021;13(7):2421. doi:
$10.3390 /$ nu13072421

6. Afshordel S, Hagl S, Werner D, Röhner N, Kögel D, Bazan NG, et al. Omega-3 polyunsaturated fatty acids improve mitochondrial dysfunction in brain aging--impact of Bcl-2 and NPD-1 like metabolites. Prostaglandins Leukot Essent Fatty Acids. 2015;92:23-31. doi: 10.1016/j.plefa.2014.05.008.

7. Lalia AZ, Dasari S, Robinson MM, Abid H, Morse DM, Klaus KA, et al. Influence of omega-3 fatty acids on skeletal muscle protein metabolism and mitochondrial bioenergetics in older adults. Aging. 2017;9(4):1096-129. doi: 10.18632/ aging.101210

8. Mc Auley MT, Mooney KM, Salcedo-Sora JE. Computational modelling folate metabolism and DNA methylation: implications for understanding health and ageing Brief Bioinform. 2018;19(2):303-17. doi: 10.1093/bib/bbw116.

9. Agodi A, Barchitta M, Quattrocchi A, Maugeri A, Canto C, Marchese AE, et al. Low fruit consumption and folate deficiency are associated with LINE-1 hypomethylation in women of a cancer-free population. Genes Nutr. 2015;10(5):480. doi: 10.1007/ s12263-015-0480-4.

10. Zarei M, Zarezadeh M, Hamedi Kalajahi F, Javanbakht MH. The Relationship Between Vitamin D and Telomere/Telomerase: A Comprehensive Review. J Frailty Aging. 2021;10(1):2-9. doi: 10.14283/jfa.2020.33.

11. Tokarz J, Möller G, Artati A, Huber S, Zeigerer A, Blaauw B, et al. Common Muscle Metabolic Signatures Highlight Arginine and Lysine Metabolism as Potential Therapeutic Targets to Combat Unhealthy Aging. Int J Mol Sci. 2021;22(15) :7958. doi: 10.3390/ijms22157958.

12. Gurău F, Baldoni S, Prattichizzo F, Espinosa E, Amenta F, Procopio AD, et al. Antisenescence compounds: A potential nutraceutical approach to healthy aging. Ageing Res Rev. 2018;46:14-31. doi: 10.1016/j.arr.2018.05.001.

13. Nargeh H, Aliabadi F, Ajami M, Pazoki-Toroudi H. Role of Polyphenols on Gut Microbiota and the Ubiquitin-Proteasome System in Neurodegenerative Diseases. J Agric Food Chem. 2021;69(22):6119-44. doi: 10.1021/acs.jafc.1c00923.

14. Lagouge M, Argmann C, Gerhart-Hines Z, Meziane H, Lerin C, Daussin F, et al Resveratrol improves mitochondrial function and protects against metabolic disease by activating SIRT1 and PGC-1alpha. Cell. 2006;127(6):1109-22. doi: 10.1016/j. cell.2006.11.013.

15. Kung H-C, Lin K-J, Kung C-T, Lin T-K. Oxidative Stress, Mitochondrial Dysfunction, and Neuroprotection of Polyphenols with Respect to Resveratrol in Parkinson's Disease. Biomedicines. 2021;9(8):918. doi: 10.3390/biomedicines9080918.

16. Abuznait AH, Qosa H, Busnena BA, El Sayed KA, Kaddoumi A. Olive-oil-derived oleocanthal enhances $\beta$-amyloid clearance as a potential neuroprotective mechanism against Alzheimer's disease: in vitro and in vivo studies. ACS Chem Neurosci. 2013;4(6):973-82. doi: 10.1021/cn400024q

17. Menendez JA, Joven J, Aragonès G, Barrajón-Catalán E, Beltrán-Debón R, BorrásLinares I, et al. Xenohormetic and anti-aging activity of secoiridoid polyphenols present in extra virgin olive oil: a new family of gerosuppressant agents. Cell Cycle Georget Tex. 2013;12(4):555-78. doi: 10.4161/cc.23756.

18. Rigacci S, Miceli C, Nediani C, Berti A, Cascella R, Pantano D, et al. Oleuropein aglycone induces autophagy via the AMPK/mTOR signalling pathway: a mechanistic insight. Oncotarget. 2015;6(34):35344-57. doi: 10.18632/oncotarget.6119.

19. Fernández del Río L, Gutiérrez-Casado E, Varela-López A, Villalba JM. Olive Oil and the Hallmarks of Aging. Mol Basel Switz. 2016;21(2):163. doi: 10.3390/ molecules21020163.

20. Peng Y, Hou C, Yang Z, Li C, Jia L, Liu J, et al. Hydroxytyrosol mildly improve cognitive function independent of APP processing in APP/PS1 mice. Mol Nutr Food Res. 2016;60(11):2331-42. doi: 10.1002/mnfr.201600332.

21. Sun W, Wang X, Hou C, Yang L, Li H, Guo J, et al. Oleuropein improves mitochondrial function to attenuate oxidative stress by activating the Nrf2 pathway in the hypothalamic paraventricular nucleus of spontaneously hypertensive rats. Neuropharmacology. 2017;113(Pt A):556-66. doi: 10.1016/j.neuropharm.2016.11.010.

22. Kumar R, Sharma A, Kumari A, Gulati A, Padwad Y, Sharma R. Epigallocatechin gallate suppresses premature senescence of preadipocytes by inhibition of PI3K/Akt/ mTOR pathway and induces senescent cell death by regulation of Bax/Bcl-2 pathway. Biogerontology. 2019;20(2):171-89. doi: 10.1007/s10522-018-9785-1.

23. Lilja S, Oldenburg J, Pointner A, Dewald L, Lerch M, Hippe B, et al. Epigallocatechin Gallate Effectively Affects Senescence and Anti-SASP via SIRT3 in 3T3-L1 Preadipocytes in Comparison with Other Bioactive Substances. Oxid Med Cell Longev. 2020;2020:4793125. doi: 10.1155/2020/4793125.

24. Gruendler R, Hippe B, Sendula Jengic V, Peterlin B, Haslberger AG. Nutraceutical Approaches of Autophagy and Neuroinflammation in Alzheimer's Disease: A Systematic Review. Mol Basel Switz. 2020;25(24). doi: 10.3390/molecules25246018.

25. Yousefzadeh MJ, Zhu Y, McGowan SJ, Angelini L, Fuhrmann-Stroissnigg H, Xu M, et al. Fisetin is a senotherapeutic that extends health and lifespan. EBioMedicine. 2018;36:18-28. doi: 10.1016/j.ebiom.2018.09.015.

26. Kovács Z, Brunner B, Ari C. Beneficial Effects of Exogenous Ketogenic Supplements on Aging Processes and Age-Related Neurodegenerative Diseases. Nutrients. 2021;13(7):2197. doi: 10.3390/nu13072197.

27. Veech RL, Bradshaw PC, Clarke K, Curtis W, Pawlosky R, King MT. Ketone bodies mimic the life span extending properties of caloric restriction. IUBMB Life. 2017;69(5):305-14. doi: 10.1002/iub.1627. 
28. Urquiaga I, Strobel P, Perez D, Martinez C, Cuevas A, Castillo O, et al. Mediterranean diet and red wine protect against oxidative damage in young volunteers. Atherosclerosis. 2010;211(2):694-9. doi: 10.1016/j.atherosclerosis.2010.04.020.

29. Saieva C, Peluso M, Palli D, Cellai F, Ceroti M, Selvi V, et al. Dietary and lifestyle determinants of malondialdehyde DNA adducts in a representative sample of the Florence City population. Mutagenesis. 2016;31(4):475-80. doi: 10.1093/mutage/ gew012.

30. Del Bo' C, Marino M, Martini D, Tucci M, Ciappellano S, Riso P, et al. Overview of Human Intervention Studies Evaluating the Impact of the Mediterranean Diet on Markers of DNA Damage. Nutrients. 2019;11(2): 391. doi: 10.3390/nu11020391.

31. Boccardi V, Esposito A, Rizzo MR, Marfella R, Barbieri M, Paolisso G. Mediterranean diet, telomere maintenance and health status among elderly. PloS One. 2013;8(4):e62781. doi: 10.1371/journal.pone.0062781.

32. Crous-Bou M, Fung TT, Prescott J, Julin B, Du M, Sun Q, et al. Mediterranean diet and telomere length in Nurses' Health Study: population based cohort study. BMJ. 2014;349:g6674. doi: 10.1136/bmj.g6674.

33. García-Calzón S, Martínez-González MA, Razquin C, Corella D, Salas-Salvadó J, Martínez JA, et al. Pro12Ala polymorphism of the PPAR $\gamma 2$ gene interacts with a mediterranean diet to prevent telomere shortening in the PREDIMEDNAVARRA randomized trial. Circ Cardiovasc Genet. 2015;8(1):91-9. doi: 10.1161/ CIRCGENETICS.114.000635.

34. Gu Y, Honig LS, Schupf N, Lee JH, Luchsinger JA, Stern Y, et al. Mediterranean diet and leukocyte telomere length in a multi-ethnic elderly population. Age Dordr Neth 2015;37(2):24. doi: 10.1007/s11357-015-9758-0.

35. Arpón A, Riezu-Boj JI, Milagro FI, Marti A, Razquin C, Martínez-González MA, et al. Adherence to Mediterranean diet is associated with methylation changes in inflammation-related genes in peripheral blood cells. J Physiol Biochem. 2016;73(3):445-55. doi: 10.1007/s13105-017-0552-6.

36. Gensous N, Garagnani P, Santoro A, Giuliani C, Ostan R, Fabbri C, et al. One-year Mediterranean diet promotes epigenetic rejuvenation with country- and sex-specific effects: a pilot study from the NU-AGE project. GeroScience. 2020;42(2):687-701. doi: 10.1007/s11357-019-00149-0.
37. Marin C, Ramirez R, Delgado-Lista J, Yubero-Serrano EM, Perez-Martinez P, Carracedo J, et al. Mediterranean diet reduces endothelial damage and improves the regenerative capacity of endothelium. Am J Clin Nutr. 2011;93(2):267-74. doi: 10.3945/ajcn.110.006866

38. Cesari F, Sofi F, Molino Lova R, Vannetti F, Pasquini G, Cecchi F, et al. Aging process, adherence to Mediterranean diet and nutritional status in a large cohort of nonagenarians: Effects on endothelial progenitor cells. Nutr Metab Cardiovasc Dis NMCD. 2018;28(1):84-90. doi: 10.1016/j.numecd.2017.09.003.

39. Mena M-P, Sacanella E, Vazquez-Agell M, Morales M, Fitó M, Escoda R, et al. Inhibition of circulating immune cell activation: a molecular antiinflammatory effect of the Mediterranean diet. Am J Clin Nutr. 2009;89(1):248-56. doi: 10.3945/ ajcn.2008.26094.

40. Casas R, Sacanella E, Urpí-Sardà M, Chiva-Blanch G, Ros E, Martínez-González M-A, et al. The effects of the mediterranean diet on biomarkers of vascular wal inflammation and plaque vulnerability in subjects with high risk for cardiovascular disease. A randomized trial. PloS One. 2014;9(6):e100084. doi: 10.1371/journal pone. 0100084

41. Sureda A, Bibiloni MDM, Julibert A, Bouzas C, Argelich E, Llompart I, et al. Adherence to the Mediterranean Diet and Inflammatory Markers. Nutrients 2018;10(1):62. doi: 10.3390/nu10010062.

42. Vellas BJ. The Geriatrician, the Primary Care Physician, Aducanumab and the FDA Decision: From Frustration to New Hope. J Nutr Health Aging. 2021;25(7):821-3. doi 10.1007/s12603-021-1657-8.

43. Azzolino D, Spolidoro GCI, Saporiti E, Luchetti C, Agostoni C, Cesari M. Musculoskeletal Changes Across the Lifespan: Nutrition and the Life-Course Approach to Prevention. Front Med. 2021;8:697954. doi: 10.3389/fmed.2021.697954.

How to cite this article: K. Virecoulon Giudici. Nutrition and the Hallmarks of Aging. J Nutr Health Aging. 2021;25(9):1039-1041, http://dx.doi.org/10.1007/s12603-021 $1686-3$ 